\title{
R-QUALITY Plan: PROPOSTA DE UMA FERRAMENTA DE APRENDIZAGEM PARA TRADUZIR E CONTROLAR REQUISITOS DE PROJETO POR MEIO DE DASHBOARD
}

Aline Cafruni Gularte (alinecgularte@gmail.com) - Universidade Federal do Rio Grande do Sul.

Márcia Elisa Echeveste (echeveste.mar@gmail.com) - Universidade Federal do Rio Grande do Sul. Istefani Carísio de Paula (istefanicpaula@gmail.com) - Universidade Federal do Rio Grande do Sul.

\section{RESUMO}

O planejamento dos requisitos durante a definição do escopo e qualidade configuram-se em subprocessos essenciais na gestão de projetos. Estes subprocessos visam assegurar que as necessidades das partes interessadas sejam identificadas e acompanhadas durante todo ciclo de vida do projeto de um produto/serviço. O uso de métodos para guiar os gestores e tangibilizar as necessidades dos stakeholders até o planejamento da qualidade é difuso e depende dos padrões adotados e da experiência dos gestores. Este artigo tem como objetivo propor um artefato que integra os subprocessos de gerenciamento do escopo e qualidade por meio da adaptação de práticas do PMBOK - Sexta edição. O propósito do artefato é traduzir e controlar a qualidade dos requisitos de projeto e foi desenvolvida por meio do Design Science Research. $\mathrm{O}$ artefato desenvolvido consistiu em uma ferramenta denominada de R-Quality Plan e a aplicação foi testada num caso de desenvolvimento de um Evento de entretenimento de grande porte. As etapas dos planos são demonstradas em um framework e foram desenvolvidas usando o pacote do R, Shiny. A contribuição prática deste trabalho é estruturar as operações de definiçãa do escopo e da qualidade por meio de um artefato utilizável em projetos e capacitação de gestores.

Palavras-chave: gestão de projetos; escopo; requisito; qualidade; dashboard 


\section{INTRODUÇÃO}

Projeto é um esforço empreendido para criar um produto, serviço ou resultado exclusivo, em um período limitado (PROJECT MANAGEMENT INSTITUTE - PMI, 2017). Os requisitos do projeto são funcionalidades que um produto ou serviço deve ter para atender às demandas ou para alcançar os objetivos dos stakeholders (PEGORARO; SAURIN; PAULA, 2013), cuja gestão e cumprimento são essenciais para o sucesso do projeto. Segundo Jiao e Chen (2006), requisitos mal definidos, os quais não refletem as reais necessidades dos clientes e partes envolvidas, por serem incompletos ou inconsistentes, estão entre as principais causas de falhas de projetos, uma vez que são a base do seu escopo.

Muitos dos erros em projetos poderiam ser evitados se as organizações dispusessem de um plano de gerenciamento de requisitos, escopo e qualidade (GREQ) bem definido, controlado, medido e aprimorado. No entanto, esses conceitos não são muito claros, dificultando a ação dos gerentes no aprimoramento de seus projetos (BLASCHEK, 2003). Uma ferramenta tradicionalmente utilizada para gerenciar os requisitos ao longo de um projeto é o Quality Function Deployment (QFD) que tem sido utilizado por longa data em diferentes situações conforme mencionado em Marx, Echeveste e Paula (2011), mas que alternativas podem ser propostas para este mesmo fim?

O Project Management body of knowledge (PMBOK®) foi desenvolvido pelo Project Management Institute (PMI), com o intuito de oferece uma base de práticas de ensino de gerenciamento de projetos, sobre a qual as organizações podem criar metodologias, políticas, procedimentos, regras, ferramentas, técnicas e fases do ciclo de vida de acordo com seus projetos (PMI, 2017). O PMBOK descreve como os planos de gerenciamento dos requisitos devem ser analisados, documentados e gerenciados do início ao fim do projeto. Porém, como é um guia e não uma metodologia, tem como limitação de mostrar "o que fazer", mas não ensinar “como fazer". Esta limitação pode ocasionar na dificuldade de adaptar os conceitos em diferentes áreas de projetos.

Neste estudo, levanta-se a hipótese de que um método didático poderá facilitar a compreensão de como gerenciar os requisitos da qualidade (GREQ) e à seguinte questão de pesquisa: "como sistematizar o GREQ de forma que facilite o entendimento e consecução dos subprocessos de definição do escopo e qualidade?”.

Assim, o objetivo é propor um artefato que integra os subprocessos de gerenciamento do escopo e qualidade por meio da adaptação de práticas do PMBOK - Sexta edição (PMI, 2017). O propósito é criar uma ferramenta de ensino, de fácil entendimento e aplicação, que 
possa traduzir e controlar a qualidade dos requisitos de projeto, a partir de métricas (indicadores), estas sendo de suma importância para a tomada de ações corretivas.

Este trabalho contribui para o ensino de gestão de projetos trazendo uma ferramenta que integra os subprocessos de escopo e qualidade. Do ponto de vista prático permite acompanhar os requisitos durante o projeto para controle e auditoria do atendimento às especificações delineadas pelo cliente ou ser usada em capacitações.

\section{REVISÃO TEÓRICA}

De acordo com o PMI (2017, p.), "Projeto é um esforço temporário empreendido para criar um produto, serviço ou resultado único", os quais são realizados para cumprir objetivos através da produção de entregas e que acabam impulsionando mudanças nas organizações. Realizado por meio de processos e subprocessos, a criação de valor de negócio é fruto da coleta, análise e controle dos requisitos de projeto. Os processos de qualidade mensuram os benefícios, tanto tangíveis quanto intangíveis, que os resultados de um projeto específico fornecem às suas partes interessadas.

A área de conhecimento que trata de requisitos pode ser descrita em duas subáreas. A Engenharia de Requisitos (ER), explora os estágios em que os requisitos são executados durante o projeto, sendo: elicitação, análise e priorização, especificação e validação (SOMMERVILLE, 2007). A subárea Gerenciamento de Requisitos (GR), inclui documentação de requisitos, armazenamento, comunicação, rastreamento e rastreabilidade de forma a permitir um gerenciamento de mudança de requisitos fácil e confiável (BRAY, 2002; SOMMERVILLE, 2007).

Uma das questões a serem estudadas é como realizar o controle dos requisitos ao longo do processo. Um método de controle dos requisitos foi proposto por Echeveste, Ribeiro e Anzanello (2005) para o controle de requisitos de projetos e produtos/serviços (KPI - Key Performance Indicator). Baseado nos autores, para viabilizar o controle durante o projeto de desenvolvimento de produto, foi desenvolvida uma função capaz de medir conjuntamente as três dimensões (custo, tempo e qualidade), monitorando o desvio de cada dimensão obtido em relação ao planejado no plano de qualidade do projeto.

A premissa é que cada característica do produto/serviço ou projeto medida em indicadores de qualidade proveniente dos requisitos deva atender a um intervalo de especificação atribuído pela equipe. O intervalo de especificação diz respeito ao valor aceitável para essa característica. 
Desta forma, a métrica proposta por Echeveste, Ribeiro e Anzanello (2005) está baseada em distâncias, considerando os desvios de cada característica do produto em relação ao respectivo alvo. As características utilizadas para avaliar o projeto podem ser medidas diretamente (quantitativas) e medidas indiretamente (características qualitativas). As características quantitativas podem ser medidas em sua unidade original, enquanto, que as características qualitativas precisam ser quantificadas através de uma escala. Como regra de decisão, todas as características devem atender seus limites individuais (intervalo de aceitação). Em outras palavras, para evitar o caráter compensatório, se houver uma característica fora do limite aceitável, o projeto deveria ser interrompido e ações corretivas devem ser tomadas.

Para operacionalizar o método proposto pelos autores, uma forma é permitir o controle automatizado em relação a softwares. O R é uma linguagem de programação utilizada para facilitar o uso e controle de processos, sendo software livre, multiplataforma e open source, proporcionando um ambiente estatístico para análise de dados e produção de gráficos (R CORE TEAM, 2020).

O RStudio é um ambiente de desenvolvimento integrado open source com uma interface mais amigável, desenvolvido para o R, com o intuito de facilitar o desenvolvimento de códigos, resultando em novos pacotes, tais como o Shiny e o ggplot2 (RSTUDIO TEAM, 2015), estes que foram utilizados para o desenvolvimento deste trabalho e com um template mais amigável.

Especificamente, o pacote Shiny é um framework de aplicações web para o RStudio. A estrutura de uma ferramenta Shiny possui dois componentes: (i) o $u i$, constrói a interface do usuário; e, (ii) o server, realiza as análises e gráficos estatísticos. As aplicações da ferramenta são interativas, podendo o usuário modificar as entradas de dados na interface de usuário e automaticamente as saídas são atualizadas (CHANG, 2017).

\section{METODOLOGIA}

Este trabalho foi baseado no Design Science Research, uma metodologia de pesquisa em tecnologia da informação baseada em resultados, que oferece diretrizes específicas para avaliação e interação em projetos de pesquisa (GEERTS, 2011). O detalhamento das etapas é apresentado a seguir (Figura 1). 


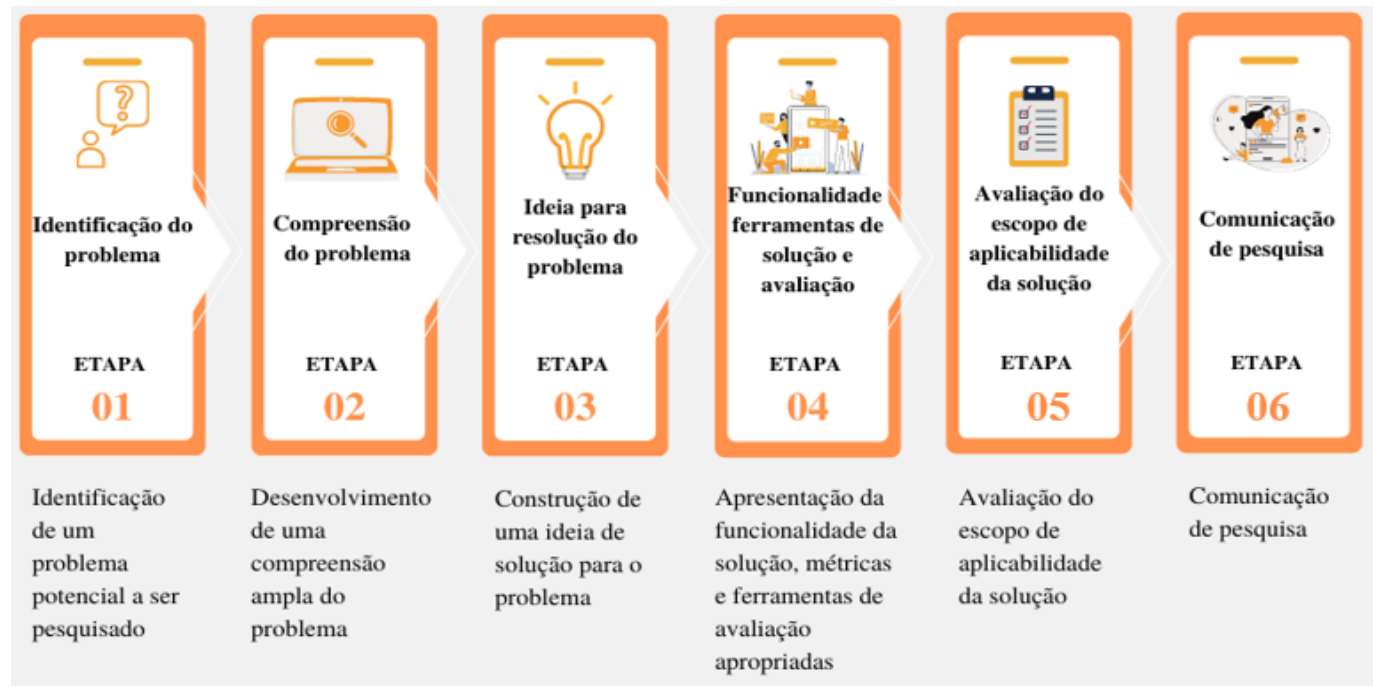

FIGURA 1 - Etapas do método de Design Science Research. Fonte: Adaptado Geerts (2011)

\subsection{Identificação do problema}

$\mathrm{Na}$ primeira etapa, identificou-se um problema potencial a ser pesquisado. Poucos estudos na literatura abordam a proposição de métodos, modelos, sistemas, plataformas e estruturas tecnológicas que ofereçam suporte ao ensino de GREQ em projetos. Young (2003) enfatiza a importância do entendimento e aplicação adequada do plano e GREQ dos projetos, para garantir a entrega de valor ao cliente. Desta forma, há a necessidade de explorar novas maneiras de representar as etapas que perpassam os planos de REQ, e de propor modelos e processos que possibilitem a um melhor entendimento desta gestão.

\subsection{Compreensão do problema}

Para a compreensão da problemática levantada na seção anterior, buscou-se na base de dados acadêmica de engenharia, Web of Science, estudos que abordassem o tema de gerenciamento de requisitos, com a string de busca ((requirements management) AND (methodology OR framework OR teaching OR instruction)). Além disso, o estudo tem como base a proposta de Echeveste, Ribeiro e Anzanello (2005). A contribuição deste artigo é operacionalizar as etapas propostas pelos autores de acordo com as etapas do framework delineadas para a GREQ por meio do pacote Shiny, do R.

\subsection{Ideia para resolução do problema}

O framework proposto integra aspectos de gestão, procedimentos pré-determinados, etapas e padronização de documentação, estabelecimento de etapas, relações internas e externas e métricas que permitem a avaliação do desempenho do processo (Figura 2). 


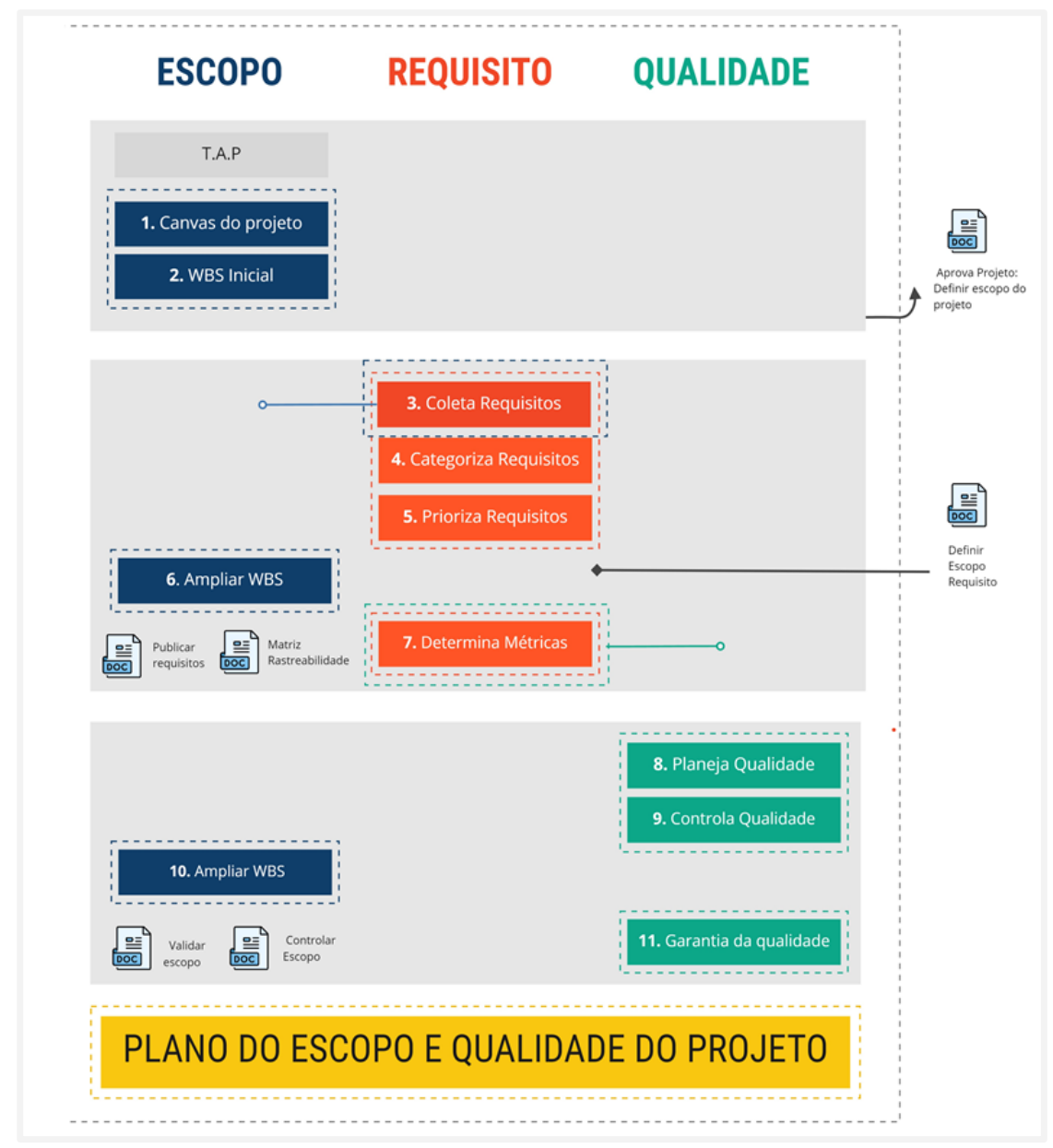

FIGURA 2 - Framework dos subprocessos de GREQ. Fonte: adaptado PMI (2017)

O framework é uma sequência de 11 etapas e 4 documentos que consolidam as decisões das etapas que o precedem. O detalhamento das etapas do framework, encontra-se no Apêndice A. A explicação detalhada das etapas é demonstrada concomitantemente ao estudo de caso na seção de resultados.

A estrutura de implementação de etapas na GREQ consiste no planejamento do escopo que compreende os requisitos os quais incluem as condições e características que devem ser atendidas pelo projeto ou devem estar presentes no produto ou serviço fornecendo dados e indicadores que comprovem a qualidade do projeto e do produto entregue (PMI, 2017).

Para consecução deste plano, são sugeridas as etapas desde o Termo de Abertura do Projeto (TAP), Canvas do projeto e a Estrutura Analítica do Projeto (EAP) (ver Figura 2).

\subsection{Funcionalidade da ferramenta de solução e avaliação}

Para facilitar a visão de todo o planejamento da GREQ, por ser um plano com diversas repartições, foi desenvolvido um artefato R-Quality Plan por meio dos pacotes ggplot2 e Shiny, 
do R. Para isso, uma aba de informações gerais apresenta uma visão mais holística de todas as etapas do framework, facilitando a navegação pelas etapas. As etapas percorreram várias rodadas de aplicação em um curso de especialização numa perspectiva project based learning $(P B L)$ no programa de pós-graduação em engenharia de produção. Os alunos seguiram as etapas do framework, ajustadas a cada projeto pelos alunos numa perspectiva $P B L$.

\subsection{Avaliação do escopo de aplicabilidade da solução}

Esta etapa consiste na apresentação da proposta de framework para avaliação de especialistas e acadêmicos. Os critérios que serão feitos para avaliar o escopo são: (i) facilidade do entendimento das ferramentas, (ii) facilidade no entendimento do modelo completo; (iii) facilidade de entender o fluxo de informação. Estes critérios foram aplicados aos alunos da disciplina de GREQ para saber o quanto a ferramenta atende esses critérios.

\subsection{Comunicação de pesquisa}

Esta etapa tem como objetivo apresentar os resultados da pesquisa ao público acadêmico de gestão de projetos. O framework proposto ajustado será difundido aos estudantes, para que possam informar quais devem ser os ajustes e melhorias a serem realizados e aos pesquisadores, gestores, que aplicaram a metodologia. Apresentar em congressos PMI, com o intuito de difundir e divulgar o estudo para obter melhorias.

\section{RESULTADOS E DISCUSSÃO}

Nesta seção são apresentadas as etapas do framework (Figura 2) com ênfase no Plano de Requisito e Qualidade operacionalizada no artefato desenvolvido em Shiny (disponível em: https://alinecgularte.shinyapps.io/appRequisitos/). Uma visão geral do app R-Quality Plan é apresentada na Figura 3.

A Figura 3 explicita a divisão das telas do artefato $R$-Quality Plan, nomeadas de: Métricas de Projetos (A) e Métricas de Produto e Serviços (B). Ambas são divididas por telas de inputs de dados (A1 e B1) e resultados (A2 e B2). 


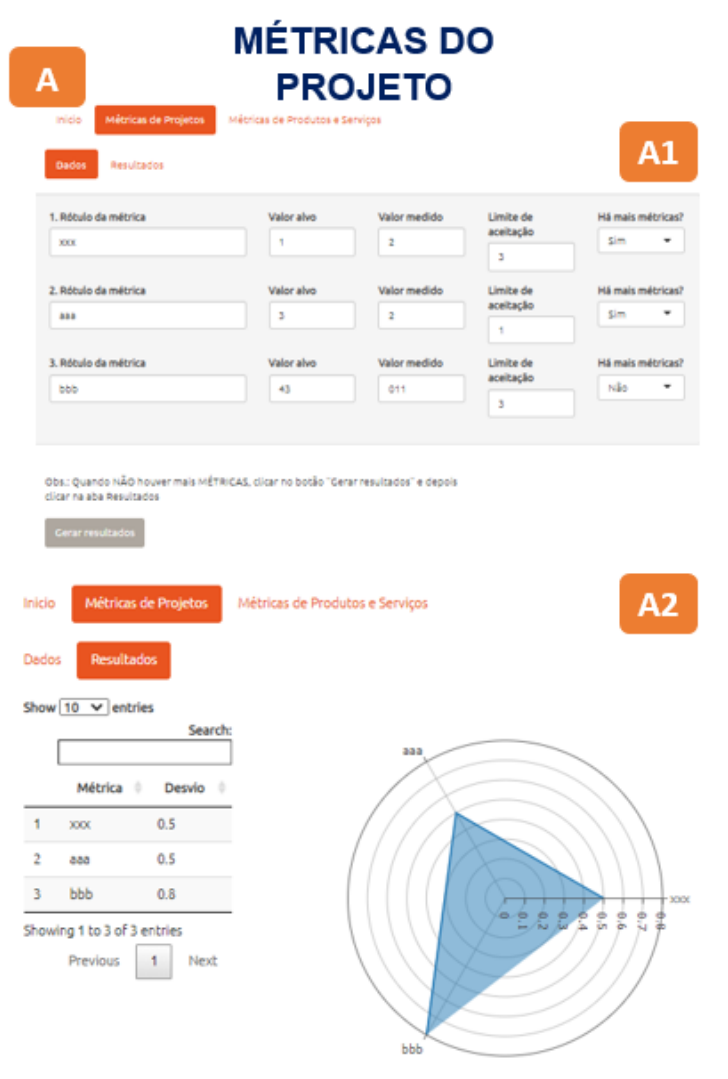

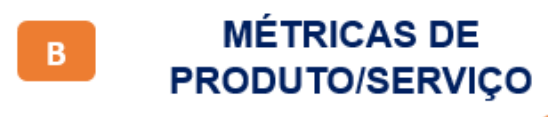
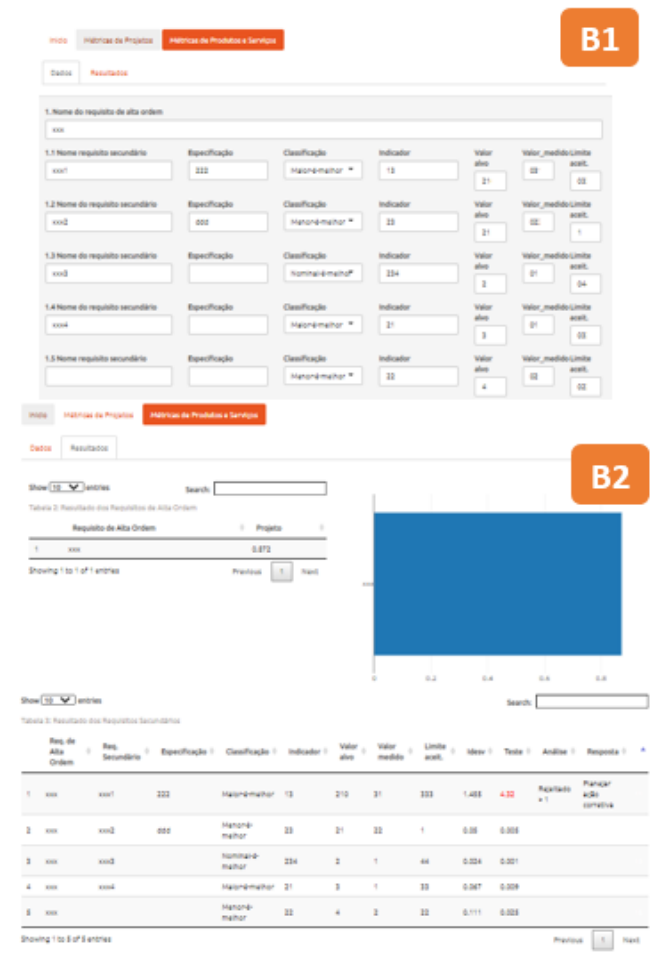

FIGURA 3 - Telas do artefato R-Quality Plan. Fonte: Autor

O detalhamento das telas do artefato será apresentado a seguir.

\subsection{Apresentação do método para um caso real na gestão de eventos}

O projeto em estudo utiliza o estudo de caso de um evento de entretenimento para celebrar o "Dia do Médico". O termo de abertura do projeto (TAP) contém informações de roteiro e cronograma das programações e layout do local do evento.

Associado aos requisitos de qualidade, as métricas de qualidade devem ser definidas e usadas para traduzir os requisitos em indicadores técnicos, mensuráveis e objetivos. Os indicadores são definidos por uma equipe multifuncional, cujos constituintes tenham familiaridade com o produto/serviço e os processos de fabricação/execução.

\subsection{Identificação e Categorização dos Requisitos de Produtos e Serviço}

Primeiramente, realizou-se o levantamento, organização e priorização dos requisitos. Neste estudo foram identificados 48 requisitos secundários (RS), os quais foram agrupados em 11 requisitos de alta ordem (RAO), sendo estes: divulgação; atração; mobiliário; som e luz; infraestrutura; decoração; gastronomia; cenografia; fotografia e filmagem; cerimonial e 
protocolo; e, suporte. Dos 48 secundários, 13 (27\%) foram considerados prioridade 1, estes que são requisitos críticos, como por exemplo "Divulgar os palestrantes do vento", requisito pertencente ao RAO “divulgação”; e, "O projeto de mobiliário deve ser compatível com as dimensões do espaço", pertencente ao RAO mobiliário. O detalhamento dos 48 requisitos é apresentado no Apêndice B.

\subsection{Identificação de Métricas para os requisitos de Projeto}

A próxima etapa foi a criação de métricas para cada um dos RS. Estas foram criadas a partir do levantamento de informações na literatura, pesquisa com especialistas e discussões em grupos. Por exemplo, para medir o RS "Divulgar os palestrantes do evento", com meta estimulada em maior ou igual a $90 \%$, contabilizou-se o $\%$ de convidados que alegaram ter conhecimento do nome dos palestrantes.

O R-Quality Plan tem como objetivo apresentar os resultados das métricas em um painel visual (dashboard), este que apresenta uma visão geral e detalhada dos requisitos, auxiliando os gestores a tomarem ações mais assertivas de seu projeto. A tela A1 (Figura 4), denominada "dados", é o campo de inclusão das seguintes informações: (i) rótulo da métrica: nome abreviado; (ii) valor alvo; (iii) valor medido; (iv) limite de aceitação. As telas a seguir foram preenchidas com o exemplo do evento "Dia do Médico".

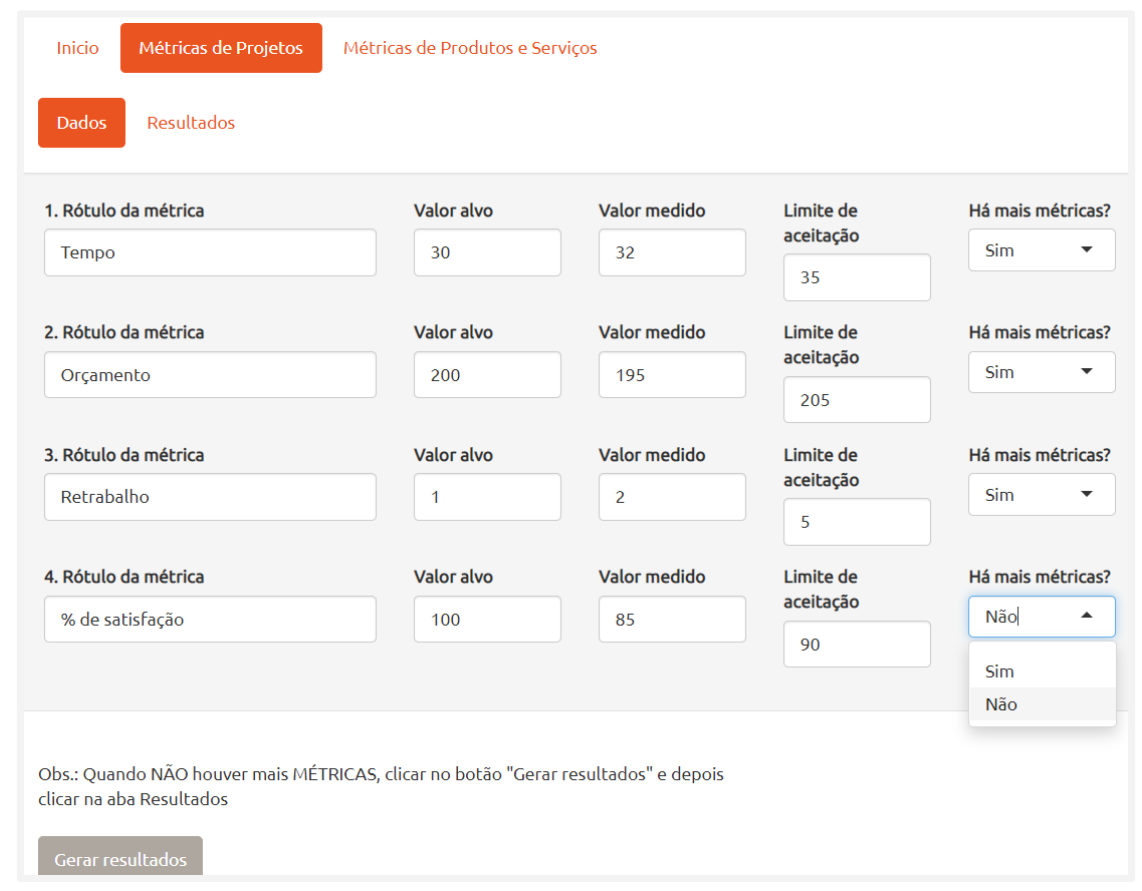

FIGURA 4 - Tela A1: entrada de dados das métricas de projetos. Fonte: Autor 
A cada campo preenchido, pergunta-se "Há mais métricas?" Caso a resposta seja "Sim", uma nova linha será incluída para que novos dados sejam preenchidos, caso assinale "Não", finaliza-se o preenchimento e clica-se no botão "Gerar resultados". O output é apresentado em "Resultados" (Tela A2), conforme Figura 5.

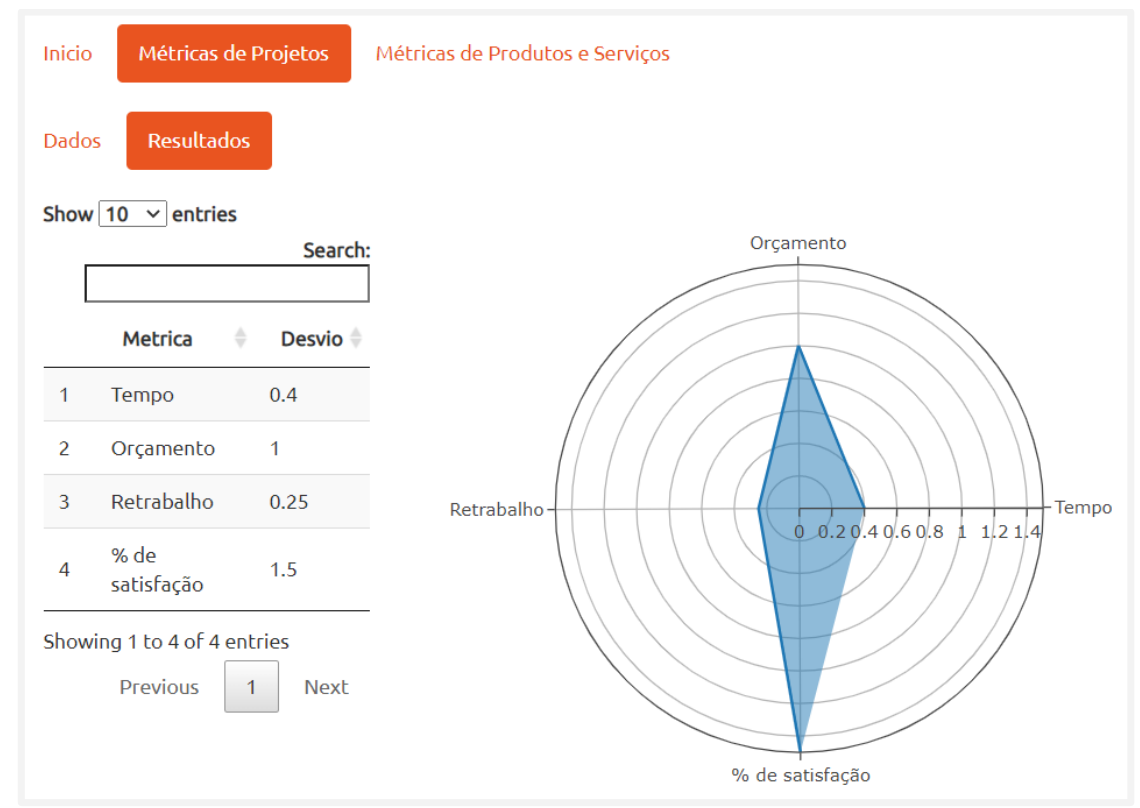

FIGURA 5 - Tela A2: dashboard das métricas. Fonte: Autor

Os cálculos dos resultados (Figura 5), são advindos da equação (1), proposta por Echeveste, Ribeiro e Anzanello (2005), conforme segue:

$$
d_{i}^{p}=\frac{\left|y_{i}^{p}-y_{i}^{0}\right|}{\left|y_{i}^{l i m}-y_{i}^{0}\right|}
$$

Sendo,

$d_{i}^{p}$ : Desvios padronizados, considerando a característica $i$ na fase $p$

$y_{i}^{p}$ : Valor medido ou atribuído para a característica $i$ na fase $p$

$y_{i}^{0}$ : Valor alvo para característica de qualidade $i$

$y_{i}^{l i m}:$ Limite de aceitação para a característica de qualidade $i$.

A equação (1), em relação à qualidade, é medida por meio da distância entre o que foi planejado e o que de fato foi executado. De acordo com esta proposta, as medidas das diversas características são, primeiramente, padronizadas para um intervalo de 0 a 1 , onde 0 coincide com o valor alvo e 1 representa o limite aceitável.

Desta forma, analisando um conjunto de características da qualidade, não se espera que todas essas características estejam abaixo do valor de aceitação. Como critério de aceitação da 
qualidade do projeto, a proposta estabelece que a média das características, convertidas para a escala 0 a 1 , deve ser igual ou inferior a 0,7 .

Assim, conforme exemplo da Figura 5, as métricas "Tempo" e "Retrabalho" estão dentro das especificações, por apresentarem desvio 0,4 e 0,25, respectivamente. A métrica “Orçamento", desvio igual a 1, está exatamente no limite de aceitação já a métrica “\% de satisfação", com desvio igual a 1,25, não atinge o alvo nem o limite de aceitação. Na Figura 5, os resultados podem ser analisados a partir da tabela e do gráfico de radar. A tela B1 é o input de dados, na qual cada RAO e suas especificações são preenchidos. A diferença entre a tela A1 e B1 é que na primeira as métricas são do projeto e na segunda, de produtos e serviços. Em B1, o usuário preenche o nome do RAO e as informações de cada um de seus RS, sendo os campos editáveis: (i) nome do requisito; (ii) especificação; (iii) classificação (nominal, maior-é-melhor, menor-é-melhor); (iv) nome do indicador; e, valores (v) alvo, (vi) medido e (vii) limite de aceitação (Figura 6).

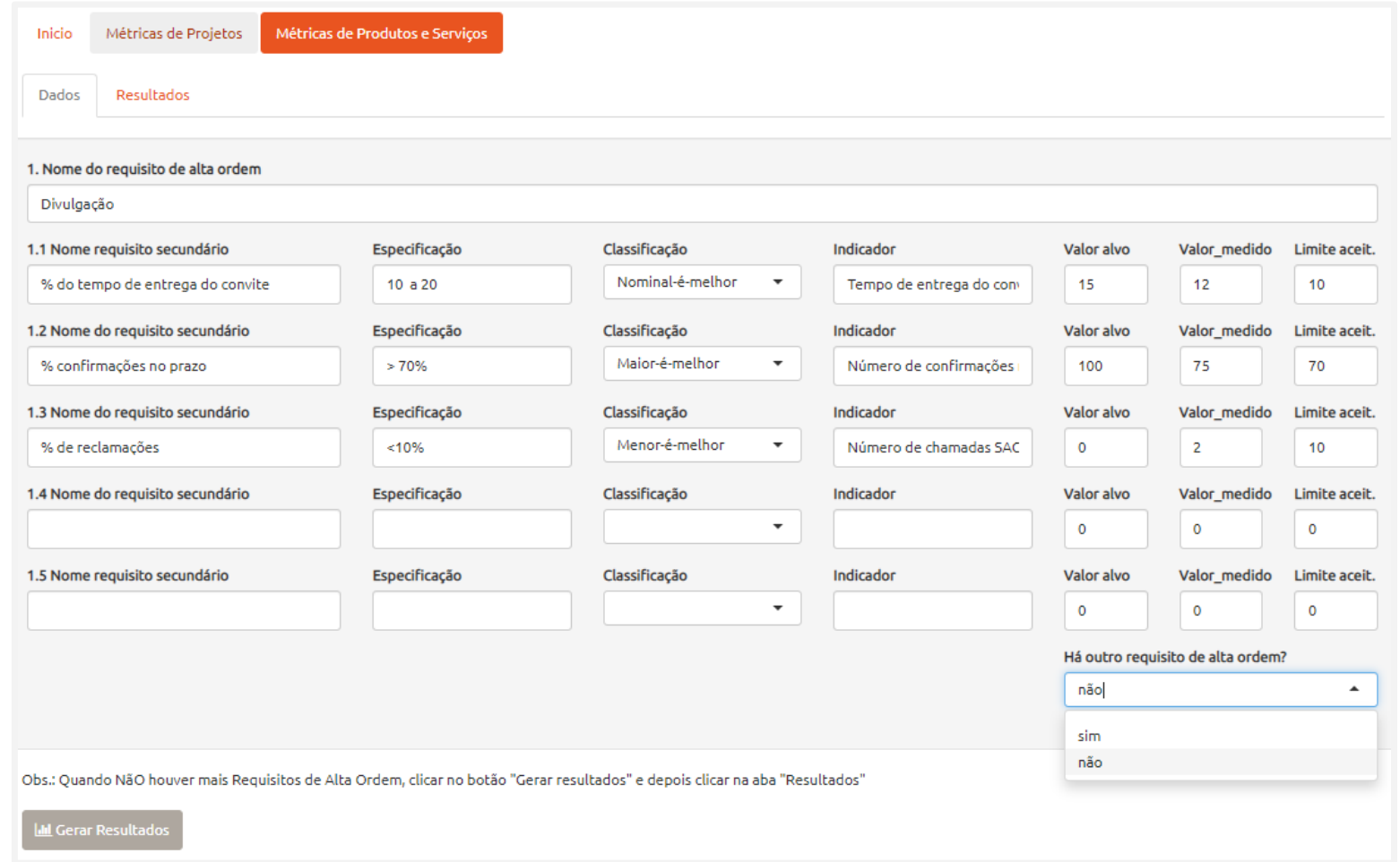

FIGURA 6 - Tela B1: entrada de dados das métricas de produtos e/ou serviços. Fonte: Autor

Ao finalizar o preenchimento das informações de todos os RS pertencente a um determinado RAO, pergunta-se "Há outro requisito de Alta Ordem?", caso a resposta seja "Sim", aparecerá uma nova linha a ser preenchida, caso "Não", finaliza-se o preenchimento e clica-se no botão "Gerar resultados". O output é apresentado em "Resultados" (Tela B2), conforme Figura 7. 


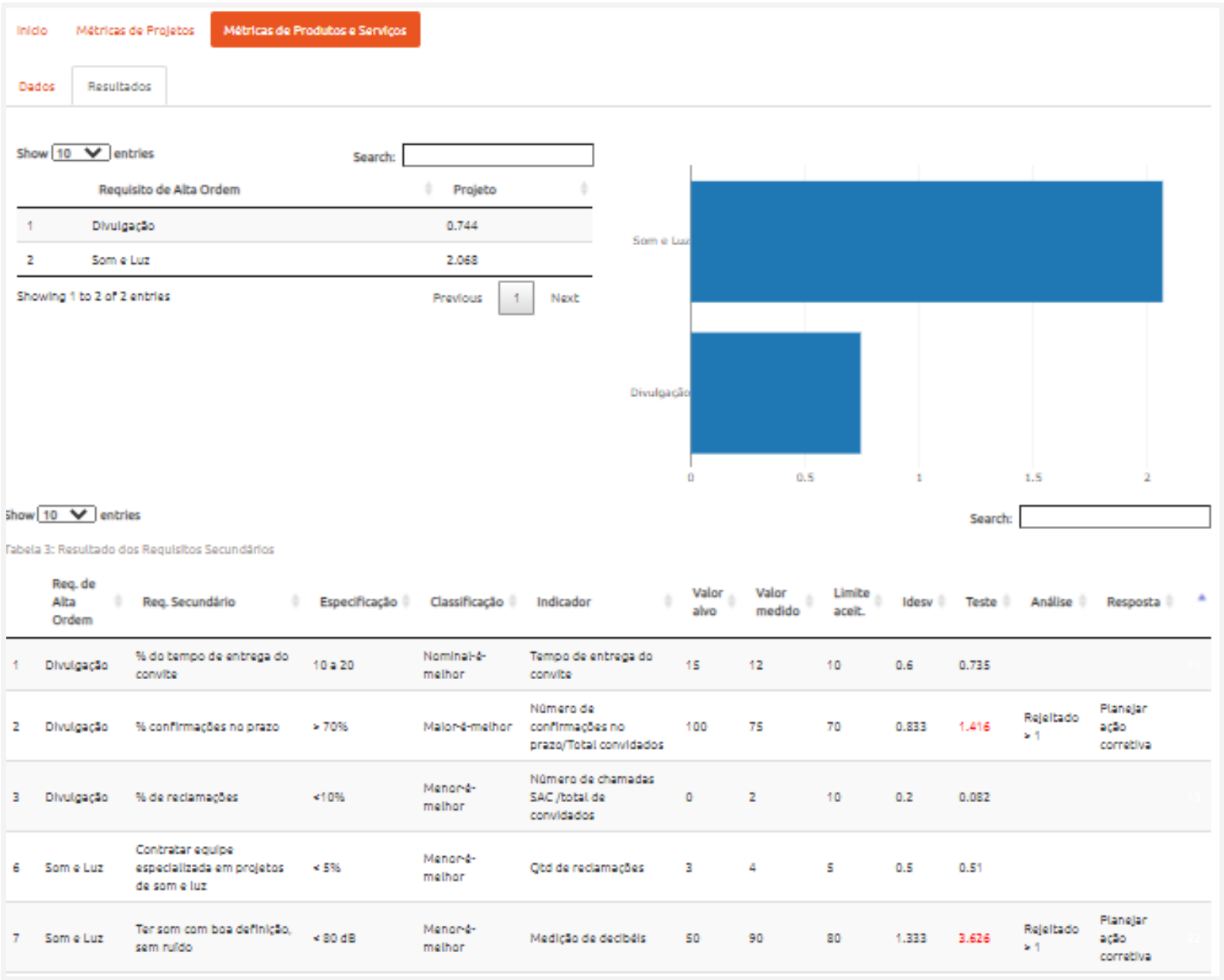

FIGURA 7 - Tela B2: dashboard das métricas de produtos e/ou serviços. Fonte: Autor

A tabela apresentada na parte inferior na Tela B2 (Figura 7), contém as seguintes informações: (i) nome do RAO; (ii) nome do RS; (iii) especificação; (iv) classificação do requisito: nominal-é-melhor, menor-é-melhor ou maior-é-melhor; (v) indicador: como é medido; valores (vi) alvo; (vii) medido; (viii) limite de aceitação; (ix) desvio (Equação 1); (x) valor do teste; (xi) análise: se o requisito é aceito ou rejeitado; caso seja rejeitado, em (xii) resposta surgirá a informação de que deverá ser planejada uma ação corretiva de melhoria no requisito.

Como exemplo ilustrativo do evento do "Dia do Médico", suponha-se que o RS de "\% de confirmações de participantes no prazo", apresente as seguintes especificações: o requisito deverá atingir um \% maior que 70\%, logo sua classificação é quanto maior-é-melhor, podendo atingir o máximo de 100\%. Para mensurar esse requisito, o indicador será o "número de participantes confirmados no prazo dividido pelo número total de participantes no evento". Assim, o valor alvo é atingir a confirmação de $100 \%$ dos convidados no prazo, com tolerância de no mínimo $70 \%$ (limite inferior). 
Considerando-se que ao contabilizar os participantes e realizar o cálculo do indicador, obteve-se que o valor atingido do requisito é igual a 75\%. Conforme as especificações estabelecidas observa-se que o valor está abaixo do valor alvo (100\%), mas acima do limite de aceitação $(70 \%)$, resultando no atendimento do requisito.

Para realizar a mesma análise de rejeição ou aceitação e mensurar a qualidade global na fase $p\left(\mathrm{Q}^{\mathrm{p}}\right)$, pertencente ao RAO, e identificar o valor do teste, deve-se calcular para cada $\mathrm{RS}$, o quadrado das distâncias elevada ao quadrado, após soma-se todos os resultados e divide-os pelo número de requisitos (n) (Equação 2).

$$
Q^{p}=\frac{1}{n} \sum_{i=1}^{n} \quad\left(\frac{d_{i}^{p}}{0,7}\right)^{2}
$$

A distância $Q^{p}$ é interpretada conforme segue ( $d_{i}^{p}$ cálculo da Equação 1 (idesv)). Medido na escala 0 a 1 , quando todas as características avaliadas apresentarem desvio $d_{i}^{p}<0,7$, resultará $Q^{p}<1$ (dentro do limite de aceitação); quando, $d_{i}^{p}=0,7$, resultará $Q^{p}=1$, (sobre o valor extremo do limite de aceitação); e, se $d_{i}^{p}>0,7$, isso deverá ser compensado por outras características próximas de zero, de modo a resultar $Q^{p}<1$; e, se houver alguma característica apresentando $d_{i}^{p}>1$, o produto não irá avançar para a próxima fase (exige reavaliação e ação corretiva). A tabela apresentada na parte superior à direita da tela (Figura 7), mostra o nome do RAO e seu respectivo valor de teste global $\left(Q^{p}\right)$.

Sendo assim, para analisar o RAO "Divulgação", que é representado pelo RS “\% de confirmações de participantes no prazo", “\% de reclamações" e "\% de entregas de convite" e sabendo que o valor do teste para esses dois últimos requisitos são de 0,735 e 0,082, respectivamente e o primeiro igual a 0,833. Aplicando a Equação 2, tem-se que o Valor de Teste Global para a "Divulgação" é de 0,744 , implicando que está dentro do limite de aceitação $\left(Q^{p}<\right.$ 1). Nota-se que o global não foi rejeitado, porém um de seus requisitos teve rejeição, sendo assim, importante analisar a tabela inferior da tela, que apresenta o resultado individual de cada requisito e acompanhar junto com o global. O resultado intermediário, está sinalizado na coluna teste da tabela.

\section{CONSIDERAÇÕES FINAIS}

Este artigo teve por propósito apresentar o desenvolvimento de um artefato que integra os subprocessos de definição do escopo e qualidade voltados para as etapas de planejamento e controle de um projeto. A proposta está alinhada com as práticas propostas no PMBOK - Sexta 
edição e pode tanto ser utilizada em capacitações quanto no gerenciamento de projetos, auxiliando gestores, professores e alunos no processo de aprendizado de GREQ.

O artefato R-Quality Plan para a análise do GREQ, fornece um template amigável, de fácil manuseio e que resulta em dashboards para monitoramento dos requisitos. $\mathrm{O}$ trabalho conclui sendo uma ferramenta adequada para transladar a informação do planejamento trazendo o método sistemático para condução da informação dos requisitos ao controle da qualidade. Além disso, a ferramenta proposta tem uma perspectiva didática que pode ser utilizada no aperfeiçoamento de gestores de projetos.

Como melhorias para trabalhos futuros é possível implementar códigos em loops para input de dados, incluindo diversos inputs e não limitando-as em 10, como neste estudo. Também, pode-se incluir todas as etapas do framework, inclusive a extração de um relatório final do projeto.

\section{REFERÊNCIAS}

BLASCHEK, J. R. Gerência de requisitos: o principal problema dos projetos de software. Rio de Janeiro: Universidade Federal do Rio de Janeiro, 2003.

BRAY, I. K. An Introduction to requirements engineering. London: Pearson Education, 2002.

CHANG, W. et al. Shiny: web application framework for R. 2017. Disponível em: https://CRAN.Rproject.org/package=shiny. Acesso em: fev. 2021.

ECHEVESTE, M. E.; RIBEIRO, J. L. D.; AnZAnELlO, M. J. A Metric system for feature and cost control during product development. Product: management \& development, v. 3, n. 2, p. 147-155, Dec. 2005.

GEERTS, G. L. A Design science research methodology and its application to accounting information systems research. International Journal of Accounting Information Systems, v. 12, n. 2, pp. 142-151, 2011.

JIAO, J. R.; CHEN, C. H. Customer requirement management in product development: a review of research issues. Concurrent Engineering: research and applications, v. 14, n. 3, pp.173-185, 2006.

MARX, A. M..; ECHEVESTE, M. E.; PAULA, I. C. Desdobramento da função qualidade aplicado ao projeto de um detergente sustentável. Production, v. 21, n. 4, p. 724-741, 2011.

PEGORARO, C.; SAURIN, T. A.; PAULA, I. C. Gestão de requisitos na construção civil: um estudo de caso focado nos requisitos ambientais de um projeto urbanístico. Produção, v. 23, n. 2, p. 345-363, 2013.

PROJECT MANAGEMENT INSTITUTE - PMI. Um Guia do conhecimento em gerenciamento de projetos (guia PMBOK). 6.ed. Newtown Square: PMI, 2017

R CORE TEAM. R: a language and environment for statistical computing. Viena: R Foundation for Statistical Computing, 2020. Disponível em: https://www.R-project.org/. Acesso em: 8 fev. 2021.

RSTUDIO TEAM. RStudio: integrated development for R. Boston: RStudio, 2015. Disponível em: http://www.rstudio.com/. Acesso em: 8 fev. 2021.

SOMMERVILLE, I. Software engineering. 8th ed. Boston: Addison-Wesley, 2007.

YOUNG, R. The Requirements engineering handbook. Norwood: Artech House, 2003. 


\section{APÊNDICE}

Apêndice A - Detalhamento das etapas do GREQ. Disponível em: Link

Apêndice B - Detalhamento dos requisitos. Disponível em: Link 